Article original

\title{
Nouveau découpage de la zone de forêt dense humide de la Côte d’Ivoire
}

\author{
N'Guessan F. KouAmé ${ }^{*}$ \& Irié A. Zoro Bi ${ }^{2}$ \\ ${ }^{1}$ Laboratoire de Botanique, UFR Biosciences, Université de Cocody, 31 BP 165 Abidjan 31, Côte d'Ivoire \\ 2 UFR Sciences de la Nature, Université d'Abobo-Adjamé, 02 BP 801 Abidjan 02, Côte d'lvoire \\ *Auteur pour les correspondances (Email: francois.kouame@univ-cocody.ci ou fnkouame@hotmail.com)
}

Reçu le 28-01-2010, accepté le 18-05-2010.

\begin{abstract}
Résumé
Les cartes de la végétation connues sur la Côte d'Ivoire sont essentiellement basées sur la physionomie de la végétation. Dans le présent travail, nous sommes partis d'une base de données floristiques récapitulant toutes les informations botaniques disponibles sur la Côte d'Ivoire, de 1882 à 2006, et riche de 46.646 saisies relatives aux taxons végétaux vasculaires de la zone de forêt dense humide ivoirienne pour établir un découpage de ladite zone. L'intégration de ces taxons et les paramètres éco-géographiques dans une analyse canonique de correspondance nous a permis d'identifier cinq principaux faciès de forêt dense humide dont quatre sont de basses altitudes. Le déterminisme de ces principaux faciès est soit géologique, soit pédologique, soit climatique, soit à la fois pédoclimatiques.
\end{abstract}

Mots clé : zone de forêt de dense, principaux faciès, déterminisme, Côte d'Ivoire

\section{Abstract}

An update of the humid forest zone splitting in Côte d'Ivoire

The vegetation maps of Côte d'lvoire are mainly based on the physionomy of vegetation. In this survey we used a botanical database of 46,646 vouchers of Ivorian vascular plants compiled from 1882 to 2006 to split up the rainforest zone. The integration of the taxa and eco-geographical parameters into a canonical correspondence analysis leads to identify 5 main features in the Ivorian rainforest area among which 4 features are lowland rainforest. The determinism of these forest features is geological, pedological, climatic or both pedo-climatic.

Key words: rainforest zone, main features, determinism, Côte d'lvoire

\section{Introduction}

La toute première carte de la forêt dense humide ivoirienne est probablement celle de Chevalier (1911) améliorée un an plus tard (Chevalier, 1912). Elle a permis à son auteur d'estimer la surface de la couverture forestière, en Côte d'Ivoire, à cette époque (Chevalier, 1920). Sur une carte de la végétation de l'Afrique Tropicale Occidentale, Roberty (1964) avait représenté la forêt dense ivoirienne à l'échelle de $1 / 1.000 .000^{\circ}$. D'autres cartes de la Côte d'Ivoire à des échelles de $1 / 200.000^{e}$ à $1 /$ $20.000^{\mathrm{e}}$ montrant la forêt dense existent (Anonyme, 1967, 1980). Mais, ce sont Guillaumet et Adjanouhoun (1968, 1969, 1971) et Guillaumet (1979) qui, ayant utilisé des photographies aériennes et le concept des unités de végétation, ont produit des cartes plus détaillées de la couverture forestière de la Côte d'Ivoire. La zone de forêt dense humide, qui correspond au Domaine guinéen sur leurs différentes cartes, est subdivisée en quatre secteurs : 1) le secteur ombrophile correspondant à la zone de forêt dont le climax principal est la forêt dense humide sempervirente ; 2) le secteur mésophile désignant la surface forestière qui a pour climax principal la forêt dense humide semi-décidue ; 3) le secteur montagnard se superposant à la zone de forêt ayant pour climax principal la forêt dense humide de montagne ; 4) le secteur littoral rassemblant toutes les portions de forêts situées à proximité de la mer et appartenant à un ensemble de climax édaphiques. Ces différents secteurs sont constitués chacun de plusieurs faciès de forêts définis par les 
associations végétales sensus Mangenot (1955). De nombreux biotopes particuliers tels que les savanes incluses, des forêts marécageuses, les forêts ripicoles, les forêts périodiquement inondées, les végétations sur les inselbergs et les bowé sont observés au sien de ces secteurs (Adjanohoun, 1964 ; Guillaumet et Adjanouhoun, 1971).

Une nouvelle carte de la végétation de la Côte d'Ivoire (Mangenot, 1971), mentionnée par certains chercheurs (de Koning, 1983 ; Riezebos et al., 1994), comporte, certainement, des différences avec les précédentes. La carte de la végétation de la Côte d'Ivoire, établie par Monnier (1983), est très proche de celles de Guillaumet et Adjanouhoun $(1968,1969,1971)$ et de Guillaumet (1979) mais en diffère par son échelle qui est plus faible, la suppression du secteur littoral et la désignation de chaque secteur par son climax principal. Elle est facilement numérisable à cause de sa faible échelle ; ce qui fait d'elle la plus utilisée pour illustrer le couvert végétal ivoirien dans les travaux scientifiques (Kouamé, 1998 ; Bakayoko, 1999 ; Kouassi, 2000).

La méthode d'inventaire de parcours de la végétation utilisée par Chevalier $(1911,1912)$ et Roberty (1964) pour établir leurs cartes de végétation, d'une part, et l'utilisation des photographies aériennes par Guillaumet et Adjanohoun (1968, 1969, 1971) et Monnier (1983) comme matériels de base d'établissement des leurs, d'autre part, sont à l'origine des différences entre leurs cartes respectives, surtout au niveau de la zone forestière de la Côte d'Ivoire. L'objetcif principal de ce travail est de vérifier la véracité de chacune de ces précédentes cartes en utilisant l'ensemble des informations botaniques disponibles en Côte d'Ivoire pour mettre en évidence les principaux faciès floristiques correspondant chacun à un type particulier de la forêt dense humide ivoirienne. L'objectif sp écifique est d'identifier les paramètres écologiques qui dominent dans la mise en place de chacun de ces types particuliers de la forêt dense humide ivoirienne.

\section{Matériel et méthodes}

\subsection{Zone d'étude}

La forêt dense humide, en Côte d'Ivoire, occupe toute la zone Sud du pays, d'Est à l'Ouest, et remonte jusqu'à $8^{\circ} 20^{\prime}$ de latitude Nord. Elle descend dans la zone centrale du pays jusqu'à $6^{\circ}$ $15^{\prime}$ de latitude Nord où la savane fait une incursion en forme de $\mathrm{V}$ communément appelé $\mathrm{V}$ Baoulé (Guillaumet et Adjanohoun, 1971). Elle est constituée de 2 types principaux de forêt dense humide climatique répondant aux définitions physionomiques de Yangambi (Trochain, 1957) ; ce sont la forêt dense humide sempervirente ou ombrophile et la forêt dense humide semi-décidue ou mésophile (Guillaumet et Adjanohoun, 1971; Guillaumet, 1979 ; Monnier, 1983).

\subsection{Extraction des données}

Le matériel d'étude est constitué par une banque de données floristiques géoréférencées dénommée SIG-Ivoire en format ACCESS ${ }^{\text {TM }}$ pour Windows, pour les tables, en formats IDRISI ${ }^{\mathrm{TM}}$ et ARCVIEW ${ }^{\mathrm{TM}}$ pour les supports cartographiques (Gautier et al., 1997 ; Chatelain et al., 2001). La base SIG-Ivoire renferme 61.164 saisies relatives à 3.780 taxons à travers 2.002 localités de récoltes couvrant l'ensemble du territoire ivoirien. Pour le présent article, il a fallu extraire de la SIG-Ivoire que les taxons dont l'aire de distribution naturelle est incluse dans la zone de forêt dense humide à travers 2 étapes successives. Les taxons savanicoles constituant, partiellement ou totalement, les flores des biotopes particuliers tels que les sommets de montagnes, les îlots de savanes, les Inselbergs et les bowé inclus dans la zone forestière ont été pris en compte.

La première extraction a consisté, d'abord, à exporter la table de saisie générale des échantillons du format ACCESS ${ }^{\mathrm{TM}}$ en format EXCEL ${ }^{\mathrm{TM}}$. Ensuite, dans le fichier EXCEL ${ }^{\mathrm{TM}}$, les données ont été triées avec, comme premier critère, la position latitudinale et, comme second critère, la position longitudinale des localités de récoltes des taxons. Ainsi, tous les taxons n'ayant été récoltés qu'au-delà de la limite Nord de la zone de forêt dense ivoirienne, située vers $8^{\circ} 20^{\prime}$ de latitude Nord, à l'Ouest et/ou à l'Est, et vers $6^{\circ} 15^{\prime}$ de latitude Nord, au Centre ont été supprimés car considérés comme exclusifs des zones de savane, en Côte d'lvoire. Le reste des données, représentant 46.646 saisies, a constitué la nouvelle base de données qui a servi à identifier les principaux faciès de la zone de forêt dense humide ivoirienne. Mais, la nouvelle base de données ainsi constituée ne peut permettre 
d'identifier, en tant qu'un ensemble unique, les faciès qui constituent la zone de forêt dense ivoirienne. L'identification de ces faciès nécessite la disponibilité de plusieurs sousensembles de localités de récoltes représentatifs des flores des différentes souszones de la forêt dense ivoirienne et offrant la possibilité de tests statistiques. Nous avons procédé, pour obtenir ces sous-ensembles de localités de récoltes, à une seconde étape d'extraction.

Celle-ci a consisté à créer, avec EXCEL ${ }^{\mathrm{TM}}$, plusieurs listes floristiques, avec les présences des taxons dans les différentes localités de récolte représentatives des forêts régionales considérées comme plus ou moins homogènes, à partir de la précédente base. Pour y parvenir, les saisies de taxons de la nouvelle base de données ont été triées par ordre alphabétique des noms de localités de récoltes. Ainsi, les localités ayant les plus grandes quantités de récoltes et dénommées localités pilotes dans cette base ont été extraites. Ces localités pilotes sont soit des entités forestières, soit des agglomérations et qui ont conféré leurs noms aux listes floristiques qu'elles ont engendrées. Les présences des taxons dans les localités avoisinantes et situées dans un rayon maximum de $50 \mathrm{~km}$ de chaque localité pilote, ont été extraites et progressivement associées à celle de la localité pilote pour former une seule liste floristique représentative de chaque forêt régionale. Cette seconde extraction nous a permis d'extraire, de la base de données floristiques de la zone de forêt dense humide de la Côte d'Ivoire, 45.178 saisies réparties entre 20 localités pilotes couvrant toutes les grandes régions de la zone de forêt dense humide ivoirienne (Tableau 1).

Pour permettre des comparaisons, tout en évitant l'effet dû aux disparités du nombre de saisies qui varie de 812, à Oumé, à 5.581, au Banco (Tableau 1 ), les données floristiques des localités pilotes ayant les fortes quantités de saisies extraites ont été subdivisées de sorte à obtenir autant de petites listes floristiques dont les présences totales des taxons avoisinent 1000. Nous avons considéré, enfin, comme sites, dans cette étude, ces petites listes provenant de la totalité ou d'une partie des listes floristiques des localités pilotes et dont les quantités totales de taxons avoisinent 1000. Ce sont 44 sites de forêt (Tableau 1), ainsi obtenus et dont les quantités de saisies varient de 812 , à
Oumé, à 1.271, à Tabou, d'une part, et les richesses floristiques oscillent entre 210 taxons, à Ayamé 2, et 908 taxons, à Grabo 2, d'autre part, qui ont servi à l'identification des principaux faciès floristiques de la zone de forêt dense humide ivoirienne.

\subsection{Analyse des données}

Différents programmes informatiques et méthodes d'analyse ont été utilisés, dans cette étude, pour atteindre les objectifs que nous nous sommes fixés. La carte a été établie avec le logiciel MAPINFO ${ }^{\mathrm{TM}}$ version 7.8 et améliorée avec le logiciel PHOTOSHOPTM version 7.0.

Les présences des taxons, dans les différents sites de forêts, et les valeurs des facteurs environnementaux des 44 sites (Tableau 2), ont été utilisées dans différents types d'analyse multivariée pour identifier les principaux faciès de la zone de forêt dense humide ivoirienne. Les données disponibles pour le type de sol et la géologie de ces sites, de nature qualitative, ont été transformées en données quantitatives sur la base du seul critère du rang qu'occupe chaque type de sol ou de géologie dans leurs classifications respectives (Perraud, 1979 ; Avenard et Tagini, 1979).

Une matrice de données floristiques, constituée de 44.295 saisies, 24.920 présences et 2.666 taxons a été obtenue en excluant de la base de données floristique de la zone de forêt dense humide ivoirienne les taxons à très faible distribution, récoltés dans un ou deux sites sur les 44 , et ceux communs à tous les 44 sites, comme le recommandent Ter Braak et Smilauer (2002) pour de telles analyses. Une seconde matrice de données, constituée de 1.659 taxons, représentant 9.943 présences pour 16.319 saisies, extraite de la précédente, a été nécessaire pour pouvoir séparer la totalité des 44 sites de forêts.

L'identification proprement dite des principaux faciès de la zone de forêt dense ivoirienne a consisté à intégrer, dans une même analyse canonique de correspondance (ACC), la matrice de données floristiques 44.295 saisies et celle des valeurs environnementales (Tableau 2). Cette étape n'a pas permis de classer 18 sites de forêt sur les 44 . La seconde matrice de données floristiques de 16.319 saisies, constituée par les 18 sites de forêt non classées précédemment, a été intégrée dans une autre ACC 
avec la même matrice des valeurs environnementales. Les deux ACC ont été réalisées avec le logiciel CANOCO pour WINDOWS ${ }^{\text {TM }}$ version 4.5 (Ter Braak et Smilauer, 2002). Le choix définitif de l'ACC, qui est du type unimodal simple, au détriment de tous les autres types d'analyse multivariée que peut permettre de réaliser CANOCO, repose sur le fait qu'elle conduit à une meilleure séparation des sites de forêts. Les données n'ont subi aucune transformation, avant les ACC successives, à cause de la faiblesse des quantités de saisies des taxons dans chaque site de forêt, variant de 0 à 67 . La présence et l'absence des espèces dans 13 forêts de basses altitudes sélectionnées dans toutes les régions ont été aussi étudiées, à travers une autre ACC, avec CANOCO pour WINDOWS ${ }^{\mathrm{TM}}$.

Pour pouvoir interpréter les résultats des ACC successives, nous avons analysé les interactions entre les différents facteurs environnementaux à travers le coefficient de corrélation de PEARSON, avec le logiciel SSPS ${ }^{\mathrm{TM}}$ version 10.0.

En vue d'étudier le poids des données floristiques dans l'existence des principaux faciès dans la zone de forêt dense humide, la matrice de 44.295 saisies, relative à tous les 44 sites, a été ensuite intégrée dans une analyse discriminante ascendante, qui est un outil d'exploration de données pour résoudre les problèmes de classification (Piélou, 1972), avec le logiciel STATISTICA ${ }^{\mathrm{TM}}$ version 6.0. Son essence principale est de séparer les objets ou évènements en groupes successifs. Le groupe de départ est graduellement divisé en des groupes de plus en plus petits. Ainsi, le degré d'association est fort entre les éléments d'un même groupe et faible entre les éléments appartenant à des groupes différents, avec une valeur allant de 0 à 1 (0 à 100 $\%)$. L'association entre les branches de chaque groupe se réfère à des mesures de la longueur rationnelle de similarité/dissimilarité.

\section{Résultats}

La projection des variables par l'analyse canonique de correspondance s'est faite sur quatre axes dont les deux premiers expriment $89,5 \%$ de la variabilité (Annexe). Les différentes étapes de l'analyse canonique de correspondance ont décelé cinq groupes de sites de forêts (Planche) correspondant chacun à un type principal de faciès de la forêt dense humide de la Côte d'Ivoire.

Le groupe 1 (sous-groupes $1 \mathrm{a}$ et $1 \mathrm{~b}$, Planche) rassemble tous les sites de forêts situés à proximité de l'Océan Atlantique. Ces sites sont, soit directement en contact avec l'Océan telles que les forêts de Bassam (Bsm1, Bsm2), de Dabou (Dbou), de Fresco (Frsc1, Frsc2), de Monogaga (Monog1, Monog2) et de Tabou (Tabou), soit séparés de l'Océan par des lagunes comme les forêts d'Adiopodoumé (Adp1, Adp2, Adp3, Adp4) et du Banco (Banco1, Banco2, Banco3, Banco4, Banco5), à l'exception de la forêt de Cavally (Cvly) qui est riveraine du fleuve Cavally. Ces sites ont leurs abscisses comprises entre 1 et 0 tandis que leurs ordonnées varient entre 0,25 et 2. Du fait de la localisation de la quasitotalité des sites de forêt de ce groupe dans la frange littorale de la forêt dense humide sempervirente, ce groupe 1 s'identifie au faciès de forêt dense humide sempervirente côtière ou littorale. Les forêts de ce faciès ont les plus faibles latitudes $\left(4,579^{\circ} \mathrm{N}\right.$ à $\left.5,467^{\circ} \mathrm{N}\right)$ mais leurs longitudes présentent une grande amplitude $\left(3,910^{\circ} \mathrm{O}\right.$ à $\left.7,130^{\circ} \mathrm{O}\right)$.

Le groupe 2 est formé des sites de forêt ayant simultanément de faibles latitudes et de faibles longitudes. II comprend les forêts d'Agboville (Agbo), d'Ayamé (Aym1, Aym2) et de Yapo (Yapo1, Yapo2, Yapo3). Les abscisses des sites de forêt de ce groupe oscillent entre $-1,2$ et 0 alors que leurs ordonnées sont comprises entre -2 et $-0,25$ (Planche). Les forêts de ce groupe sont du type sempervirent à l'exception de celle d'Agboville, qui est une transition entre le type sempervirent et le type semi-décidu (Tableau 1). Ce groupe 2 correspond au faciès de la forêt dense humide sempervirente du Sud-Est de la Côte d'Ivoire.

Le groupe 3 rassemble les sites de forêt de latitudes moyennes ou fortes et présentant une grande amplitude de longitudes. II est constitué des forêts de Divo, du Haut-Sassandra (Hsass1, Hsass2), d'Oumé (Oume) et de la Marahoué (Marah) appartenant toutes au type de forêt dense humide semi-décidue; de ce fait, ce groupe est identifié comme le faciès de la forêt dense humide semi-décidue. Les sites de forêt de ce groupe ont leurs abscisses comprises entre 0,5 et 1 tandis que leurs ordonnées varient entre 0,2 et 0,3 (Planche). 
Tableau 1: Sites de forêts issus de la seconde extraction de SIG-Ivoire et ayant servi à identifier les principaux faciès de la forêt dense humide ivoirienne

\begin{tabular}{|c|c|c|c|c|c|}
\hline Régions & Localités pilotes & Sites de forêt & Nombres de récoltes & $\begin{array}{c}\text { Nombres de } \\
\text { taxons }\end{array}$ & Types de forêt \\
\hline Est & Abengourou & Abng & 952 & 568 & Semi-décidue \\
\hline Côtière & Banco & Adp1 & 1.056 & 441 & Sempervirente \\
\hline$"$ & $"$ & Adp2 & 1.043 & 611 & $"$ \\
\hline$"$ & $"$ & Adp3 & 1.019 & 553 & $"$ \\
\hline$"$ & $"$ & Adp4 & 1.061 & 478 & $"$ \\
\hline$"$ & $"$ & Banco1 & 1.112 & 516 & $"$ \\
\hline$"$ & $"$ & Banco2 & 1.118 & 539 & $"$ \\
\hline$"$ & $"$ & Banco3 & 1.119 & 384 & $"$ \\
\hline$"$ & $"$ & Banco4 & 1.122 & 482 & $"$ \\
\hline$"$ & $"$ & Banco5 & 1.110 & 493 & $"$ \\
\hline$"$ & $"$ & Bsm1 & 907 & 404 & $"$ \\
\hline$"$ & $"$ & Bsm2 & 900 & 490 & $"$ \\
\hline Agboville & Agboville & Agbo & 928 & 533 & Transition \\
\hline Sud-Est & Ayamé & Aym1 & 1.069 & 525 & Sempervirente \\
\hline$"$ & $"$ & Aym2 & 1.017 & 210 & $"$ \\
\hline$"$ & $"$ & Aym3 & 1.008 & 904 & $"$ \\
\hline Sud-Ouest & Cavally & Cvly & 1.150 & 414 & $"$ \\
\hline Côtière & Dabou & Dbou & 1.223 & 799 & $"$ \\
\hline Sud & Divo & Divo & 841 & 463 & Semi-décidue \\
\hline Ouest & Danané & Dnn1 & 1.009 & 613 & Sempervirente \\
\hline$"$ & $"$ & Dnn2 & 1.020 & 713 & $"$ \\
\hline Côtière & Fresco & Frs1 & 819 & 743 & $"$ \\
\hline$"$ & $"$ & Frs2 & 825 & 708 & $"$ \\
\hline Centre-Ouest & Guiglo & Gglo & 1.246 & 590 & $"$ \\
\hline Sud-Ouest & Grabo & Grb1 & 1.054 & 517 & $"$ \\
\hline$"$ & $"$ & Grb2 & 1.071 & 908 & $"$ \\
\hline Centre-Ouest & Haut-Sassandra & Hsass 1 & 1.143 & 644 & Sempervirente \\
\hline$"$ & $"$ & Hsass2 & 1.081 & 878 & $"$ \\
\hline Ouest & Man & Man1 & 920 & 477 & Transition \\
\hline$"$ & $"$ & Man2 & 915 & 568 & $"$ \\
\hline Sud-Est & Maféré & Mfre & 1.042 & 606 & Sempervirente \\
\hline Centre-Ouest & Marahoué & Marah & 846 & 581 & Semi-décidue \\
\hline Côtière & Monogaga & Monog1 & 989 & 753 & $"$ \\
\hline$"$ & $"$ & Monog2 & 993 & 642 & $"$ \\
\hline Centre & Oumé & Oume & 812 & 502 & Semi-décidue \\
\hline Sud-Ouest & Soubré & Sbre & 1.325 & 603 & Sempervirente \\
\hline$"$ & Taï & Tainp1 & 1.090 & 609 & $"$ \\
\hline$"$ & $"$ & Tainp2 & 1.103 & 702 & $"$ \\
\hline$"$ & $"$ & Tainp3 & 1.065 & 606 & $"$ \\
\hline$"$ & $"$ & Tainp4 & 1.123 & 812 & $"$ \\
\hline Côtière & Tabou & Tabou & 1.271 & 706 & $"$ \\
\hline Sud-Est & Yapo & Yapo1 & 878 & 485 & $"$ \\
\hline$"$ & $"$ & Yapo2 & 902 & 482 & $"$ \\
\hline$"$ & $"$ & Yapo3 & 881 & 492 & $"$ \\
\hline
\end{tabular}



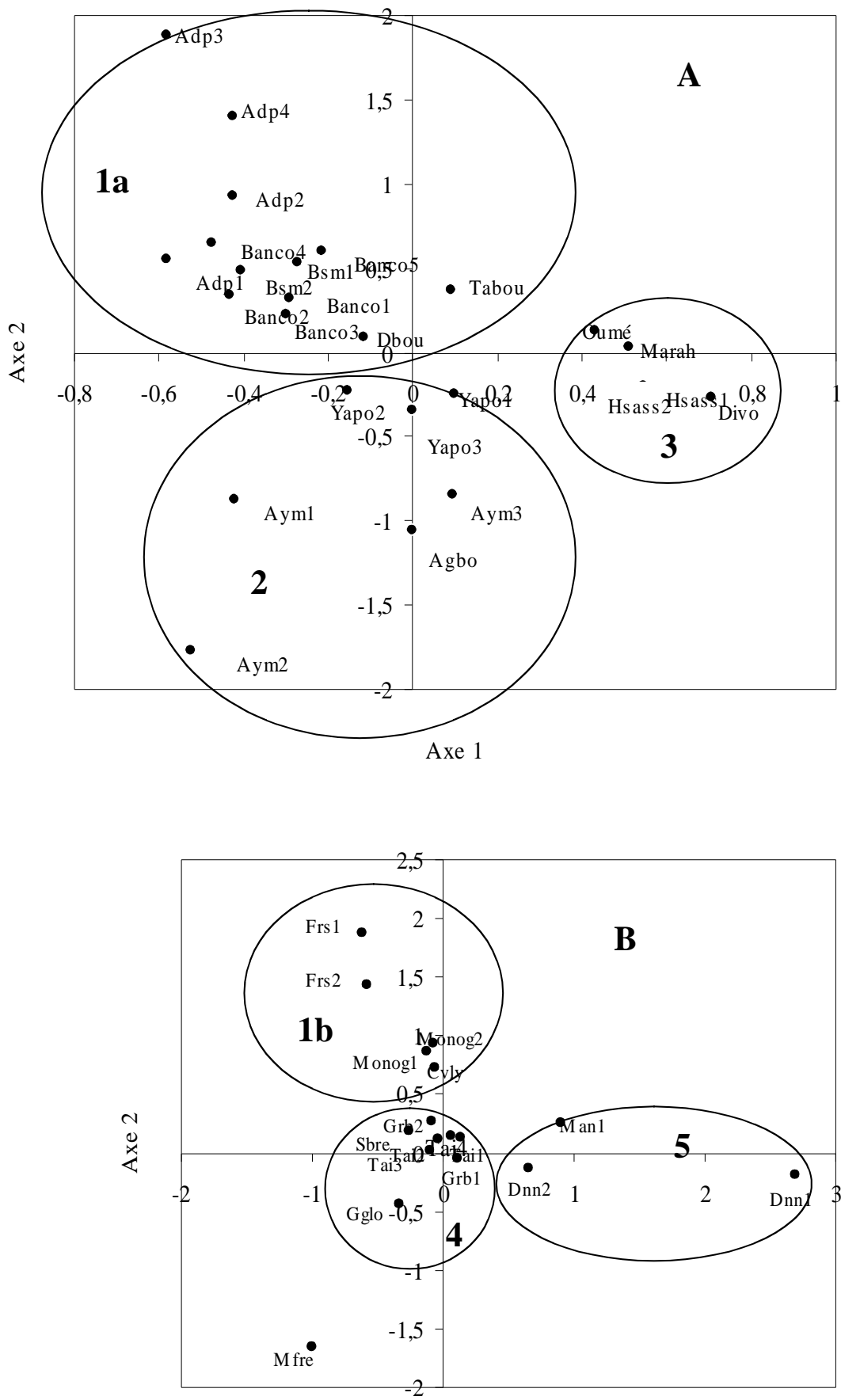

Axe 1

Planche : Ordination des sites de forêt par Analyses Canoniques de Correspondance successives, dans le plan formé par les axes 1 et 2

En A les 26 sites ayant été séparés par la premère ACC et en B les 18 sites qui ont nécessité une seconde ACC pour être séparés.

Sci. Nat. Vol.7 No2 : 177 - 194 (2010) 
Le groupe 4 , indistinct après la première étape de l'Analyse Canonique (Planche), unit les sites de forêt de Guiglo (Gglo), de Grabo (Grb1, Grb2), de Soubré (Sbre) et de Taï (Tainp1, Tainp2, Tainp3, Tainp4), qui sont toutes de faibles latitudes et de fortes longitudes (Tableau 2). Les abscisses de ce groupe sont comprises entre 0,5 et 0,3 (Planche) alors que les ordonnées sont dans l'intervalle $[-0,7 ; 0,4]$. Les forêts de ce groupe appartiennent toutes au type de forêt dense humide sempervirente et définissent le faciès de forêt dense humide sempervirente du Sud-Ouest de la Côte d'Ivoire.

Le groupe 5, également indistinct après la première étape de l'Analyse Canonique, rassemble les sites de forêt de Danané (Dnn2) et de Man (Man1, Man2) dont la particularité est leur localisation aux voisinages de $7,38^{\circ} \mathrm{N}$. Les cordonnées de ce groupe sont inscrites dans les intervalles $[0,7 ; 2,7]$, pour les abscisses, et $[-0,9 ; 0,8]$, pour les ordonnées (Planche). Ce groupe correpond au faciès de la forêt de montagne, à cheval sur le type de forêt dense humide sempervirente et la transition avec le type de forêt dense humide semi-décidue (Tableau 2). Les sites de forêt de ce groupe sont tous de fortes latitudes et de longitudes comprises entre 6,85 et $7,79^{\circ} \mathrm{O}$.

En dehors de ces cinq groupes de forêts plus ou moins homogènes, trois sites de forêts s'individualisent. Ainsi, le site de la forêt de Maféré (Mfre), indistinct après la première étape de l'Analyse Canonique, s'isole dans la seconde étape de cette analyse (Planche $B$ ); mais ses cordonnées, dans le plan formé par les axes 1 et 2 , l'intègrent dans le groupe 2 . Le second site de la forêt de Danané (Dnn1) est plus proche du groupe 4 que de celui du groupe 5 mais n'intègre aucun de ces deux groupes. Le site de la forêt d'Abengourou (Abgn), appartenant au type de forêt semi-décidue et dont l'abscisse est égale à 7 , n'a finalement pas été pris en compte dans la constitution des groupes parce qu'il n'aurait pas permis une bonne lisibilité de ceux-ci.

L'analyse discriminante ascendante, basée sur le coefficient de Pearson couplé au coefficient de similitude de Sørensen, confirme ces cinq groupes de forêt (Fig. 1) dans leurs grands traits, même si une homogénéité parfaite n'est pas revélée à l'intérieur de chaque groupe. Le groupe 1 rassemble 10 sites au palier $\mathrm{I}_{3}$ et 2 sites, respectivement, aux paliers $\mathrm{J}_{2}$ et $\mathrm{N}_{3}$; les sites de Dabou (Dbou) et de Bassam 1 (Bsm1) ne sont pas directement liés aux autres sites de ce groupe. Le palier $\mathrm{H}_{1}$ isole les 3 sites de forêt de Yapo (Yapo) comme caractéristiques de la forêt dense humide sempervirente du Sud-Est ivoirien et les éloigne, de peu, des sites de forêt d'Agboville et de Maféré. La forêt dense humide sempervirente du Sud-Ouest est caractérisée par les paliers $J_{1}$ et $M_{1}$; les autres sites de Grabo (Grb1, Grb2) et de Soubré (Sbre) sont dispersés par l'analyse. Une similarité de $71 \%$ est observée entre le site Grb2 et un des sites d'Ayamé (Aym3) ; ceci traduit une très grande ressemblance floristique entre ces 2 sites. Tous les sites de forêt de montagne confluent au palier $\mathrm{B}_{1}$. Les sites de forêt dense humide semi-décidue se retrouvent au sein de 2 triplets représentés par les paliers $B_{2}$ et $D_{2}$, très distants sur l'axe des ordonnées du dendrogramme (Fig. 1) ; le triplet $B_{2}$ est proche des sites de forêt de montagne.

Au niveau des 13 forêts de basses altitudes sélectionnées, 4 groupes de forêts se distinguent dans le plan formé par les axes 1 et 2 de l'analyse canonique de correspondance (Fig. 2). Le groupe I, constitué par les forêts semi-décidues (Bossématié, Haut Sassandra, Marahoué), se caractérise par des valeurs élevées du déficit hydrique (Fig. 3) et de la position latitudinale (Fig. 4). Les forêts sempervirentes côtières (Banco, Dassioko, Port Gauthier, Monogaga) forment le groupe II, qui est caractérisé par une faible position latitudinale et des précipitations moyennes sur un sol appartenant au groupe de sables tertiaires (Fig. 3). Le groupe III est constitué par les forêts sempervirentes du Sud-Ouest (Haute-Dodo, Taï) tandis que le groupe IV correspond à l'ensemble des forêts sempervirentes du Sud-Est (Mabi, Songan, Tamin, Yaya). Ces 2 derniers groupes se caractérisent par une position latitudinale moyenne, des précipitations plus ou moins élevées et un faible déficit hydrique (Fig. 3). Ils diffèrent fondamentalement du type de leurs soussols. Les forêts de Haute-Dodo et de Taï reposent sur un sous-sol essentiellement granitique alors que celles de Mabi, Songan, Tamin, Yaya recouvrent un sous-sol schisteux. Le déficit hydrique annuel et la position latitudinale sont les paramètres les plus fortement corrélés à l'axe 1 (Fig. 3), avec lequel ils ont une forte corrélation positive $(r=0,70 ; P<0,01 ; N=13)$. L'axe 2 a une forte corrélation positive $(r=0,90 ; P<0,01 ; N=$ 13) avec le sol sableux tertiaire, une forte corrélation négative $(r=-0,80 ; P<0,01 ; N=13)$ avec le soussol schisteux et une faible corrélation négative $(r=$ 0,$61 ; P<0,05 ; N=13$ ) avec la latitude (Fig. 4). 
Tableau 2 : Données environnementales et situations géographiques des sites de forêt utilisées dans les analyses multivariées

\begin{tabular}{|c|c|c|c|c|c|c|c|c|}
\hline Sites & $\begin{array}{l}\text { Altitude } \\
\text { (m) }\end{array}$ & $\begin{array}{c}\text { Déficit } \\
\text { hydrique } \\
(\mathrm{mm})\end{array}$ & $\begin{array}{l}\text { Durée de la } \\
\text { sécheresse } \\
\text { (mois) }\end{array}$ & $\begin{array}{c}\text { Géologie } \\
\text { (ordre) }\end{array}$ & $\begin{array}{l}\text { Précipitations } \\
\text { annuelles } \\
(\mathrm{mm})\end{array}$ & $\begin{array}{l}\text { Type } \\
\text { de sol } \\
\text { (ordre) }\end{array}$ & $\begin{array}{l}\text { Latitude } \\
\quad\left({ }^{\circ}\right)\end{array}$ & $\begin{array}{c}\text { Longitude } \\
\left({ }^{\circ}\right)\end{array}$ \\
\hline Abng & 200 & 460 & 5 & 2 & 1.350 & 5 & 6,180 & $-3,595$ \\
\hline Adp1 & 75 & 300 & 4 & 0 & 2.050 & 1 & 5,432 & $-4,120$ \\
\hline Adp2 & 75 & 300 & 4 & 0 & 2.050 & 1 & 5,432 & $-4,120$ \\
\hline Adp3 & 75 & 300 & 4 & 0 & 2.050 & 1 & 5,432 & $-4,120$ \\
\hline Adp4 & 75 & 300 & 4 & 0 & 2.050 & 1 & 5,432 & $-4,120$ \\
\hline Agbo & 75 & 350 & 4,5 & 2 & 1.550 & 18 & 6,250 & $-3,858$ \\
\hline Aym1 & 150 & 260 & 4 & 2 & 1.910 & 2 & 5,571 & $-3,560$ \\
\hline Aym2 & 150 & 260 & 4 & 2 & 1.910 & 2 & 5,571 & $-3,560$ \\
\hline Aym3 & 150 & 260 & 4 & 2 & 1.910 & 2 & 5,571 & $-3,560$ \\
\hline Banco1 & 75 & 300 & 4 & 0 & 2.120 & 1 & 5,467 & $-3,910$ \\
\hline Banco2 & 75 & 300 & 4 & 0 & 2.120 & 1 & 5,467 & $-3,910$ \\
\hline Banco3 & 75 & 300 & 4 & 0 & 2.120 & 1 & 5,467 & $-3,910$ \\
\hline Banco4 & 75 & 300 & 4 & 0 & 2.120 & 1 & 5,467 & $-3,910$ \\
\hline Banco5 & 75 & 300 & 4 & 0 & 2.120 & 1 & 5,467 & $-3,910$ \\
\hline Bsm1 & 25 & 300 & 4 & 0 & 2.100 & 1 & 5,415 & $-3,840$ \\
\hline Bsm2 & 25 & 300 & 4 & 0 & 2.100 & 1 & 5,415 & $-3,840$ \\
\hline Cvly & 450 & 275 & 4 & 1 & 1.950 & 2 & 5,763 & $-7,043$ \\
\hline Dbou & 50 & 325 & 4 & 0 & 1.950 & 1 & 5,328 & $-4,260$ \\
\hline Divo & 300 & 230 & 3 & 1 & 1.605 & 18 & 5,658 & $-4,855$ \\
\hline Dnn1 & 1.300 & 280 & 4 & 1 & 2.200 & 4 & 7,381 & $-7,795$ \\
\hline Dnn2 & 1.300 & 280 & 4 & 1 & 2.200 & 4 & 7,381 & $-7,795$ \\
\hline Frs1 & 25 & 400 & 5 & 2 & 1.650 & 3 & 5,310 & $-5,660$ \\
\hline Frs2 & 25 & 400 & 5 & 2 & 1.650 & 3 & 5,310 & $-5,660$ \\
\hline Gglo & 300 & 300 & 4 & 2 & 1.720 & 2 & 6,511 & $-7,235$ \\
\hline Grb1 & 400 & 100 & 1,5 & 1 & 2.400 & 4 & 5,328 & $-6,815$ \\
\hline Grb2 & 400 & 100 & 1,5 & 1 & 2.400 & 4 & 5,328 & $-6,815$ \\
\hline Hsass 1 & 300 & 350 & 5 & 1 & 1.600 & 10 & 7,103 & $-6,360$ \\
\hline Hsass2 & 300 & 350 & 5 & 1 & 1.600 & 10 & 7,103 & $-6,360$ \\
\hline Man1 & 1.200 & 350 & 5 & 4 & 1.800 & 2 & 7,381 & $-6,850$ \\
\hline Man2 & 1.200 & 350 & 5 & 4 & 1.800 & 2 & 7,381 & $-6,850$ \\
\hline Mfre & 200 & 250 & 4 & 2 & 1.950 & 2 & 5,693 & $-3,525$ \\
\hline Monog1 & 25 & 375 & 5 & 1 & 1.750 & 5 & 5,084 & $-5,905$ \\
\hline Monog2 & 25 & 375 & 5 & 1 & 1.750 & 5 & 5,084 & $-5,905$ \\
\hline Marah & 400 & 325 & 5 & 2 & 1.350 & 13 & 6,946 & $-5,748$ \\
\hline Oume & 400 & 280 & 4 & 3 & 1.350 & 17 & 6,494 & $-5,765$ \\
\hline Sbre & 100 & 250 & 4 & 1 & 1.700 & 20 & 5,954 & $-6,518$ \\
\hline Tainp1 & 200 & 250 & 3 & 1 & 2.100 & 4 & 5,502 & $-6,833$ \\
\hline Tainp2 & 200 & 250 & 3 & 1 & 2.100 & 4 & 5,502 & $-6,833$ \\
\hline Tainp3 & 200 & 250 & 3 & 1 & 2.100 & 4 & 5,502 & $-6,833$ \\
\hline Tainp4 & 200 & 250 & 3 & 1 & 2.100 & 4 & 5,502 & $-6,833$ \\
\hline Tabou & 25 & 250 & 3 & 1 & 2.300 & 2 & 4,579 & $-7,130$ \\
\hline Yapo1 & 100 & 280 & 4 & 2 & 1.950 & 3 & 5,624 & $-3,998$ \\
\hline Yapo2 & 100 & 280 & 4 & 2 & 1.950 & 3 & 5,624 & $-3,998$ \\
\hline Yapo3 & 100 & 280 & 4 & 2 & 1.950 & 3 & 5,624 & $-3,998$ \\
\hline
\end{tabular}




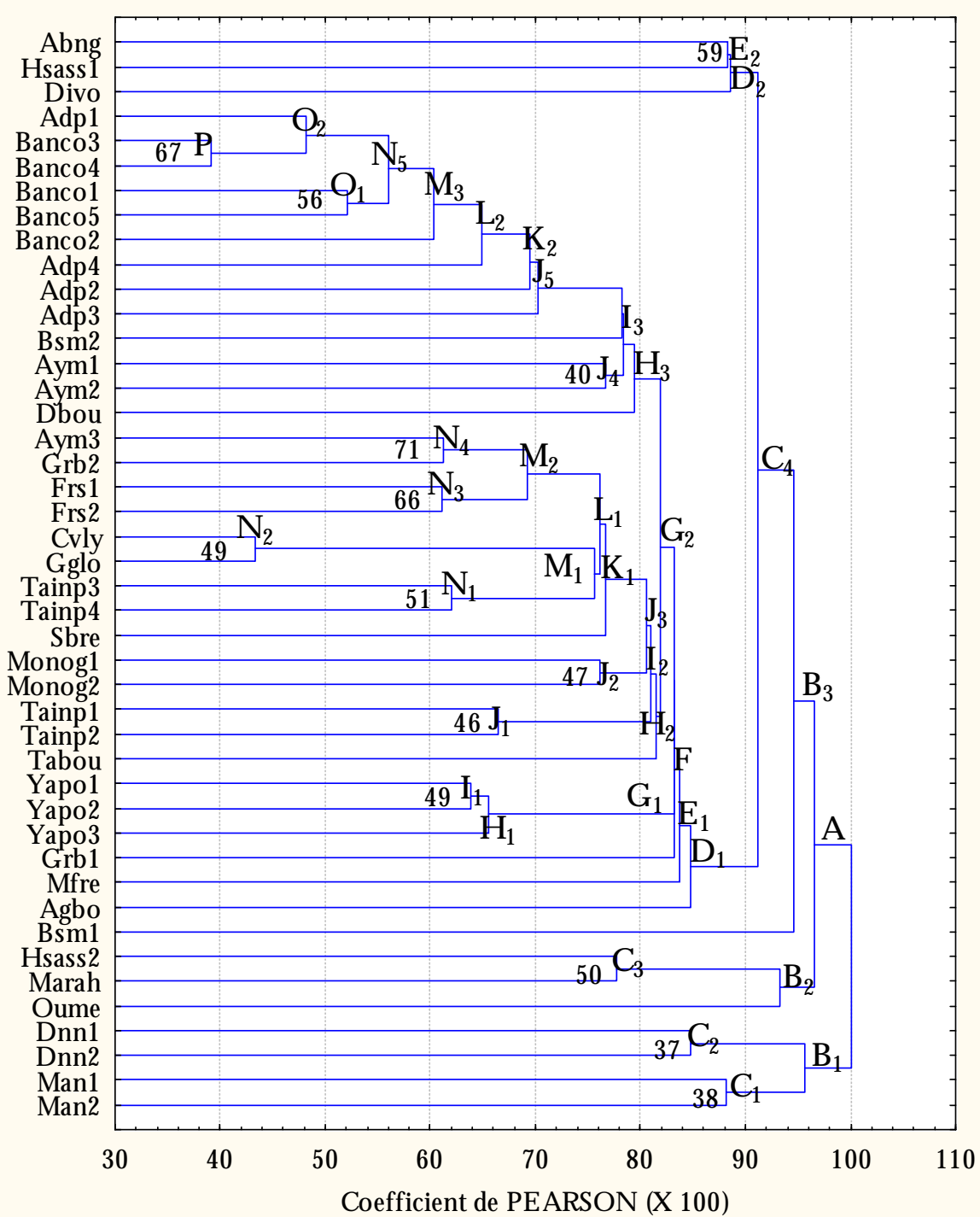

Figure 1: Dendrogramme des sites de forêt suivant leurs coefficients de Pearson

Les coefficients de Pearson, traduisant les interactions entre les sites, sont en abscisses. Les noms des sites, abrégés ou entiers et souvent accompagnés de chiffres de 1 à 5, sont en ordonnées. Les différents paliers sont désignés dans le dendrogramme pardes lettres de l'alphabet de $A$ à $P$. Les chiffres arabes aux niveaux des différents paliers expriment l'indice de similarité de Sørensen entre les sites de forêt. Pour les noms de sites, se référer au Tableau 1. 


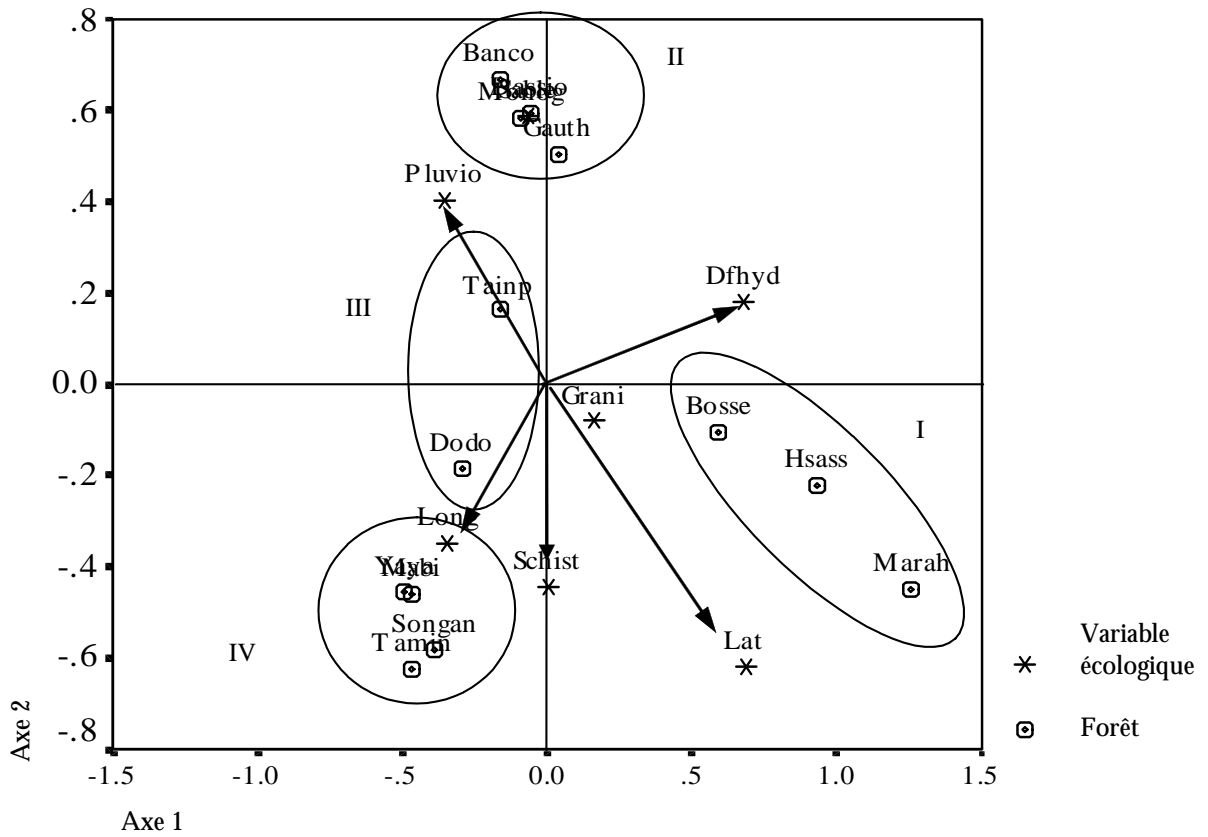

Figure 2 : Ordination des forêts de basses altitudes sélectionnées dans le plan formé par les axes 1 et 2 d'après l'analyse canonique de correspondance

Le plan formé par les axes 1 et 2 exprime $31 \%$ de la variabilité totale des espèces et $44 \%$ de celle des variables environnementales. Les flèches indiquent les variables écologiques. Les noms abrégés des forêts : Hsass = Haut-Sassndra, Bosse = Bossématié, Marah = Marahoué, Dodo = Haute Dodo, Gauth $=$ Port Gauthier, Monog = Monogaga, Dassio = Dassioko, Tain $=$ Taï. Les noms abrégés des variables écologiques $:$ Dfhyd $=$ Déficit hydrique, Grani $=$ Granite, Lat $=$ Latitude, Long $=$ Longitude, Pluvio $=$ Pluviométrie annuelle, Schist $=$ Schiste.

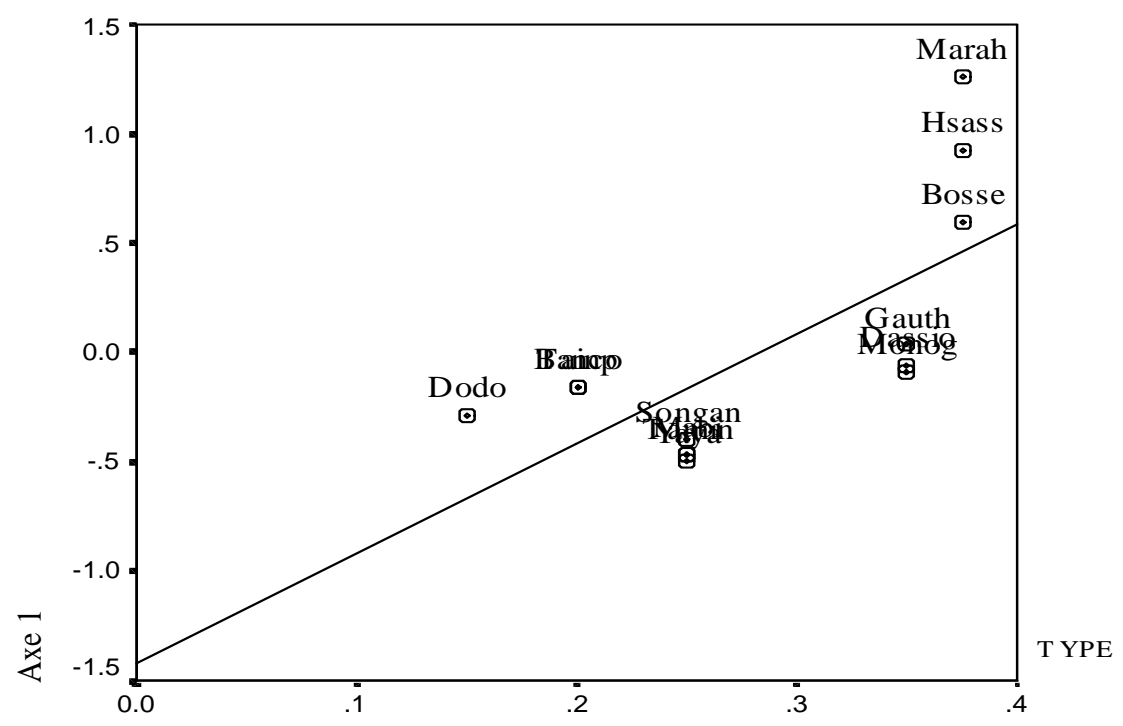

Déficit hydrique $(\mathrm{mm})$

Figure 3 : Ordination des 13 forêts de basses altitudes sélectionnées dans le plan formé par l'axe 1 et le déficit hydrique. Les noms abrégés des forêts $:$ Hsass = Haut-Sassndra, Bosse = Bossématié, Marah = Marahoué, Dodo = Haute Dodo, Gauth = Port Gauthier, Monog = Monogaga, Dassio = Dassioko, Tain = Taï. 


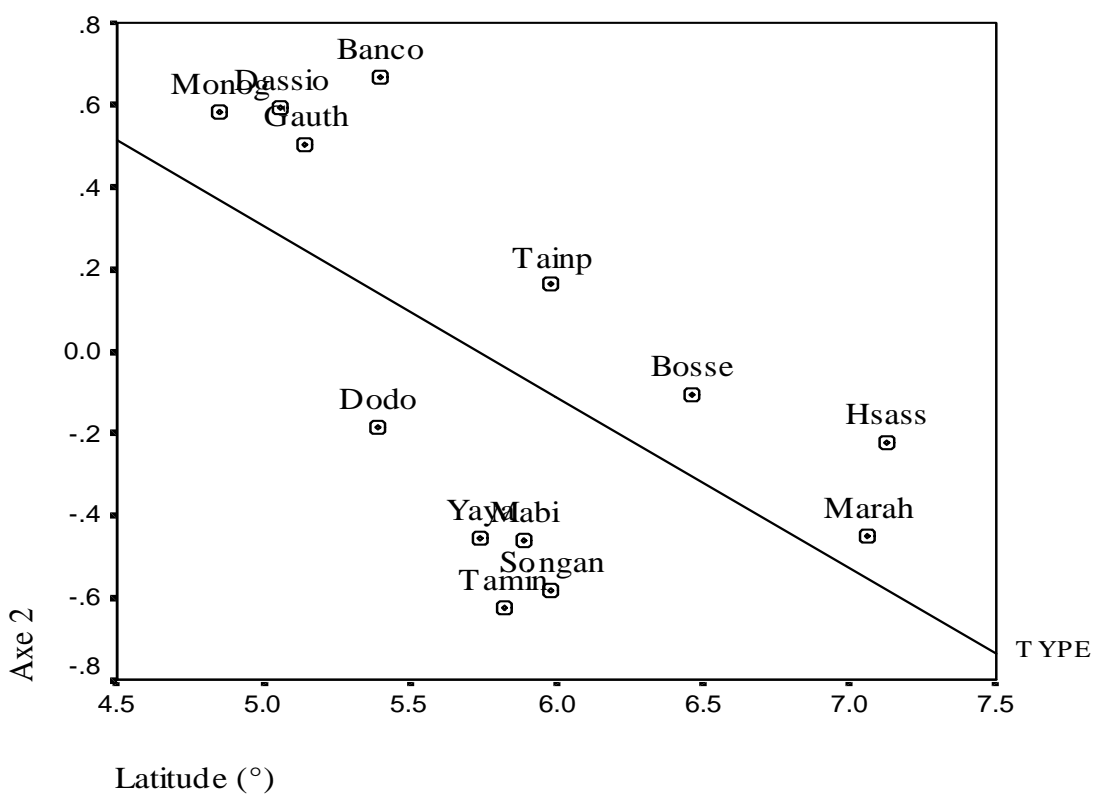

Figure 4 : Ordination des 13 forêts de basses altitudes sélectionnées dans le plan formé par l'axe 2 et la latitude. Les noms abrégés des forêts : Hsass = Haut-Sassndra, Bosse = Bossématié, Marah = Marahoué, Dodo = Haute Dodo, Gauth = Port Gauthier, Monog = Monogaga, Dassio = Dassioko, Tain = Taï.

\section{Discussion}

Les cinq principaux faciès floristiques identifiés dans la zone de la forêt dense humide ivoirienne (Fig. 5), à travers les analyses canoniques (Planche, Fig. 2) et l'analyse discriminante ascendante (Fig. 1), trouvent leurs explications dans les conditions écologiques (Figs. 3 et 4 ) et historiques des différentes régions de ladite zone. Chaque faciès floristique est le résultat de l'hégémonie d'un groupe de facteurs par rapport aux autres groupes de facteurs.

Les facteurs climatiques sont variables dans la zone de forêt dense humide, en fonction de la latitude, de la longitude et de l'altitude (Eldin, 1971). L'évapotranspiration potentielle, le nombre et la durée de la saison sèche croissent avec la latitude tandis que les précipitations et la durée de la saison humide diminuent, malgré les ondulations des isohyètes par rapport à la latitude (Kouamé, 1998). Le nombre de saisons humides diminue avec la longitude. Indépendamment des autres groupes de facteurs écologiques, les facteurs climatiques sont responsables de l'existence de 3 des principaux faciès floristiques de la zone de forêt dense humide ivoirienne. En effet, les forêts denses humides sempervirentes du Sud-Est, celles du Sud-Ouest et les forêts denses humides semi-décidues intègrent les forêts climatiques de Guillaumet et Adjanohoun (1971). Ces forêts sont situées généralement à moins de $1000 \mathrm{~m}$ d'altitude ; leur existence semble dépendre essentiellement de deux facteurs climatiques simultanés: un total pluviométrique annuel de plus $1.350 \mathrm{~mm}$ et une saison sèche ne dépassant pas trois à quatre mois annuellement (Anonyme, 1983 ; Richards, 1996 ; Blanc, 2002).

Si nous pouvons admettre, à la suite de Guillaumet et Adjanohoun (1971), que les facteurs climatiques expliquent la séparation des forêts denses humides sempervirentes des forêts denses humides semi-décidues, ce groupe de facteurs ne peut expliquer l'existence des deux faciès au niveau des forêts sempervirentes. En effet, ces deux faciès, ayant les mêmes facteurs climatiques et identifiés comme des centres de gradients de diversités floristiques en Côte d'Ivoire (Hall et Swaine, 1981 ; Sayer et al., 1992 ; Kouamé et al., 2004) doivent leur séparation aux facteurs géologiques qui diffèrent dans les deux régions. La zone de forêt 
dense humide ivoirienne est essentiellement représentée par des roches éruptives et des sédiments métamorphiques, généralement, désignés sous le nom de birrimien (Lamotte et Rougerie, 1952a et 1952b). Le substratum de ces glacis est séparé en deux blocs Est et Ouest, dans la région de Tiassalé, par le V-Baoulé (Dabin et al., 1960 ; Avenard et Tagini, 1979). Dans le bloc forestier occidental, il est essentiellement sous la forme granitique, rarement sous la forme schisteuse ou de roches vertes. Dans le bloc forestier oriental, il est invariablement sous forme de schistes birrimiens.

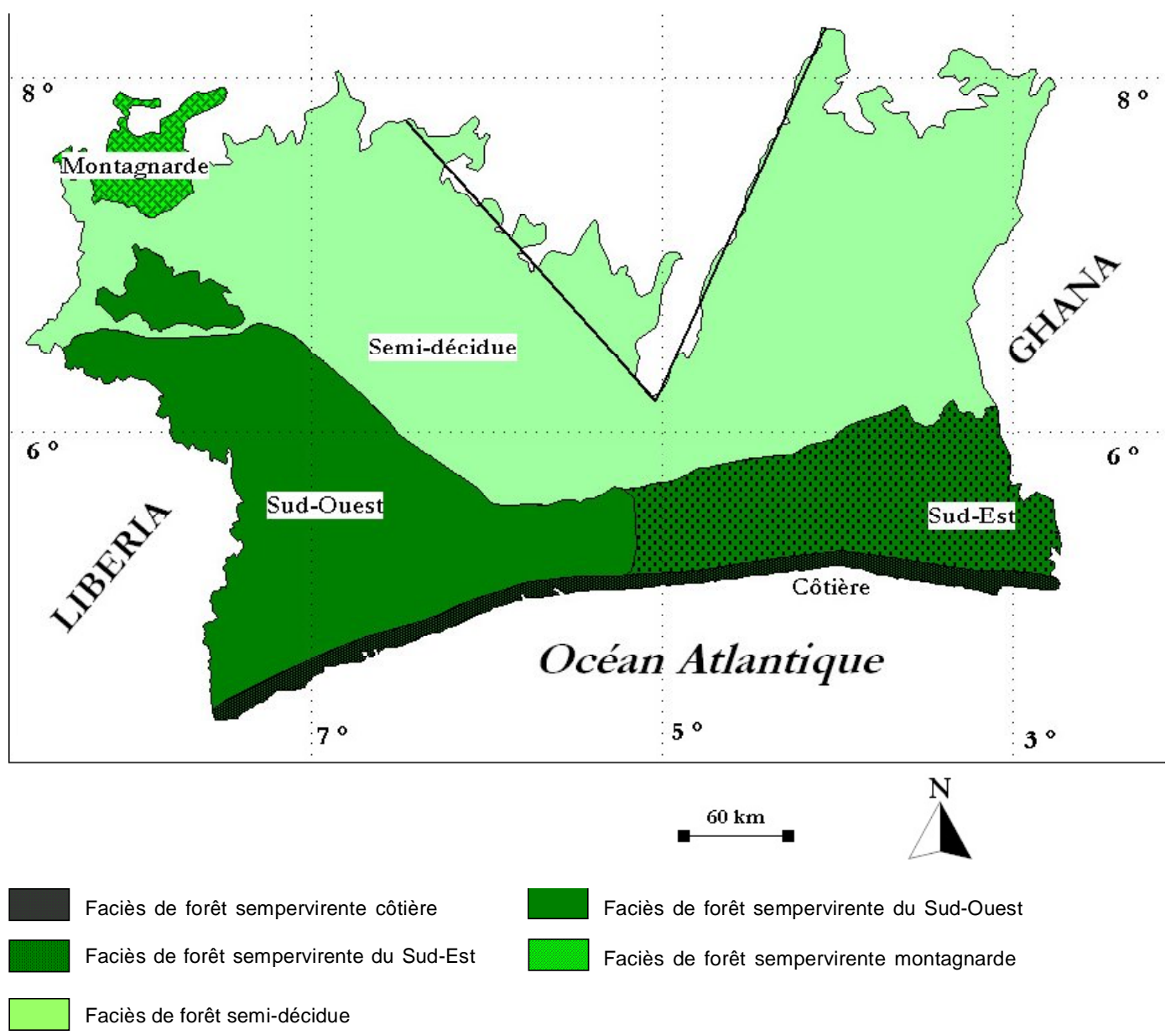

Figure 5 : Carte de distribution potentielle des principaux faciès floristiques de la zone de forêt dense humide de Côte d'Ivoire

Sur le plan géologique, la frange de l'Ouest ivoirien, constituée d'un socle épilibérien issu de la cratonisation des chaînes libériennes, formant le secteur de la semi-plateforme éburnéenne (Avenard et Tagini, 1979), crée des conditions favorables à l'existence d'un faciès floristique lié à l'altitude. Ainsi la région de Man, avec les Monts Nimba culminant à $1.750 \mathrm{~m}$ d'altitude et correspondant à la retombée orientale de la dorsale guinéenne (Lamotte et Rougerie, 1952a et 1952b ; Rougerie et Lamotte, 1952), est l'endroit des plus hauts sommets de la zone forestière de la Côte d'Ivoire (Avenard, 1971 ; Avenard et Tagini, 1979). Cette région serait un lambeau du vieux socle constitué de granites à hypersthène, qui est resté protégé de la migmatisation générale de l'Ouest africain (Avenard, 1971). Le type de formations végétales rencontré dans cette 
région correspond au faciès de forêts montagnardes, dénommé secteur montagnard par Guillaumet et Adjanohoun (1971) et correspondant à l'unique centre de gradient de diversité floristique altitudinal, en Côte d'Ivoire (Sayer et al., 1992 ; Van Rompaey, 1993). Guillaumet et Adjanohoun (1971) ont attribué la cause de ce faciès à un climat dû à l'altitude.

Au niveau de la pédologie, les deux sous-classes et tous les seize groupes des sols ferrallitiques sont représentés dans le sous-bois forestier ivoirien (Perraud, 1979). La zone littorale est caractérisée par des sols ferrallitiques fortement désaturés et appauvris (Dabin et al., 1960) ou remaniés sur lesquels se développent des formations végétales particulières dont le groupe de sol constitue le principal déterminisme. Cette zone est le lieu de prédilection de la forêt sempervirente côtière correspondant au secteur littoral de Guillaumet et Adjanohoun (1971) identifié par ces auteurs comme un ensemble de plusieurs climax édaphiques dans lequel il n'y a pas de climax dominant. Mais la zone littorale, telle que définie dans notre étude, prend en compte des entités de forêts telles que les forêts d'Adiopodoumé, de Banco, de Dassioko, de Monogaga etc., plus ou moins grandes et ayant certaines de leurs parties éloignées d'une dizaine de kilomètres de la côte Atlantique. De ce fait, la forêt sempervirente devient le climax dominant, de par l'étendue de son aire de distribution potentielle, de cette zone. Toutefois, les faibles variations de l'altitude et des facteurs climatiques, dans cette zone (Tableau 2), conduisent à l'intégration de ces forêts denses humides sempervirentes côtières au groupe de forêts édaphiques tropicales africaines constituées par les forêts marécageuses, les mangroves, les forêts périodiquement inondées, les forêts ripicoles, les forêts sur sables, les forêts sur sols argileux et les savanes édaphiques.

L'existence de différents faciès dans la zone de forêt dense humide ivoirienne est connue depuis Mangenot (1955) ; mais il revient à Guillaumet (1967 et 1979), Guillaumet et Adjanohoun (1971) de les avoir véritablement étudiés. Ces auteurs se sont basés, d'abord, sur l'endémisme de certains taxons végétaux qui, en Côte d'Ivoire, sont confinés entre le fleuve Sassandra et la frontière ivoiro-libérienne ; d'où l'appellation d'espèces sassandriennes pour désigner ce groupe de taxons et de faciès sassandrien pour nommer le type de forêt de cette région (Mangenot, 1956). Mais il y eut cinq sous-groupes d'espèces sassandriennes dont le plus large correspondrait à la sous-région d'endémisme Ouest africaine ou Haute Guinée (Guillaumet, 1967 ; Guillaumet et Adjanohoun, 1971 ; Poorter et al., 2004). Différents groupements forestiers climatiques ont été aussi identifiés sur la base de la conception d'association de Mangenot (1955) et désignés par une combinaison des noms des espèces ou des genres les plus abondantes dans chaque groupement. Mais la plupart de ces groupements présente des chevauchements (Guillaumet et Adjanohoun, 1971). Ce qui laissait présager l'existence, dans les régions où il y avait ces chevauchements, de plus grands ensembles de végétation ayant un maximum de cortège floristique en commun, eu-égard aux conditions écologiques très voisines ou communes, et dans lesquels s'intégreraient plusieurs de ces petits groupements forestiers.

Les cinq principaux faciès identifiés dans ce travail (Fig. 5) recoupent, en bien de points, certains des quatre secteurs du Domaine guinéen (Guillaumet et Adjanohoun, 1971 ; Monnier, 1983), en Côte d'Ivoire ; ils sont d'ailleurs très proches des différents types principaux de forêt dense humide identifiés au Ghana (Hall et Swaine, 1981). La différence fondamentale avec les types de forêt dense humide connus en Côte d'Ivoire réside en la séparation du secteur ombrophile de Guillaumet et Adjanohoun (1971) en deux faciès sempervirents dont l'un appartient au Sud-Est et l'autre au Sud-Ouest ivoirien. La forêt sempervirente de basses altitudes de Monnier (1983) se disloque en trois faciès dont les deux précédents et un faciès côtier longeant le littoral. Le faciès de forêt sempervirente du Sud-Ouest correspond aux deux premiers groupes d'espèces sassandriennes de Guillaumet (1967), responsables de l'existence du gradient de diversité floristique dans le SudOuest de la Côte d'Ivoire. Le gradient de diversité floristique reconnu dans le Sud-Est ivoirien est engendré par le faciès de forêt sempervirente du Sud-Est. Ces deux faciès de forêts sempervirentes du Sud-Est et du Sud-Ouest correspondent aux forêts pélophiles ou forêts caractérisées par l'association Diospyro-Mapanietum de Mangenot (1955). Ces groupes de forêts exigent une grande quantité d'eau utilisable pour les espèces végétales et ne peuvent se développer sur un sol pauvre en argile (Guillaumet, 1967). Les forêts 
psammophiles ou forêts caractérisées par l'association Thurraeantho-Heisterietum de Mangenot (1955), qui ont une préférence pour les sols à faible capacité de rétention en eau et pauvres en argile, constituent le faciès de forêt sempervirente côtière ou littorale. Le faciès de forêt dense semi-décidue identifié dans ce travail se superpose au groupe de forêts à Celtis spp et Triplochiton scleroxylon Schumann dénommé successivement Triplochitetalia (Schnell, 1950) et Celtidetalia (Mangenot, 1955). De même, le faciès de forêt montagnarde que nous avons identifié correspond à la forêt montagnarde de Monnier (1983).

\section{Conclusion}

Les résultats de nos travaux ont révélé l'existence, dans la zone de forêt dense humide ivoirienne, de cinq principaux faciès floristiques (Planche, Fig. 5) dont quatre sont de basses altitudes et un de hautes altitudes. Ces faciès correspondent, pour certains, à des secteurs de forêt sur les cartes de la végétation connues sur la Côte d'Ivoire. Ainsi, le faciès de la forêt dense semidécidue correspond-il au secteur de la forêt dense semi-décidue caractérisée par le genre Celtis L. et Triplochiton scleroxylon Schumann; celui de la forêt montagnarde se superpose au Secteur montagnard dû aux plus hautes altitudes du pays. Mais, avec nos travaux, le Secteur de la forêt dense sempervirente ou ombrophile devient en réalité un ensemble hétérogène constitué de trois principaux faciès floristiques. Le faciès de la forêt sempervirente côtière ou littorale occupe toute la zone Sud de la Côte d'Ivoire en contact, soit direct avec l'Océan Atlantique, soit indirect en passant par les lagunes et correspond aux forêts psammophiles caractérisées par l'association Turraeantho-Heisterietum. Le Sud-Est et le SudOuest de la Côte d'Ivoire, identifiés comme centres de gradients floristiques se prolongeant, respectivement au Ghana et au Libéria, correspondent chacun à un faciès de forêt dense humide sempervirente; ce sont les forêts pélophiles caractérisés par l'association Diospyro-Mapanietum.

Le déterminisme de ces principaux faciès est soit géologique, soit pédologique, soit climatique, soit à la fois pédo-climatiques. En effet, le faciès de la forêt dense sempervirente montagnarde est d'origine géologique. Le faciès de la forêt dense sempervirente littorale ou côtière, dont la particularité est du fait de sa présence, au niveau du cordon littoral isolant les systèmes lagunaires de l'Océan et constitués de sédiments quaternaires, est d'origine pédologique. Celui de la forêt dense semidécidue, invariablement représenté de part et d'autre du V-Baoulé, n'est régi que par les faibles précipitations et leurs corollaires de longue saison sèche et de déficit hydrique élevé; de ce fait, il est déterminé uniquement par le climat. Ceux des forêts sempervirentes du Sud-Est et du Sud-Ouest correspondent aux foyers des précipitations de basses altitudes en Côte d'Ivoire et diffèrent essentiellement du point de vue pédologique. Par conséquent, leur déterminisme est à la fois pédologique et climatique.

\section{Remerciements}

Nous sommes reconnaissants aux Conservatoire et Jardin botaniques de Genève pour la mise à disposition de la base de données utilisée dans ce travail. Nous remercions la Coopération Française pour avoir financé 9 mois de séjour à Paris pour renforcer la base de données et le Muséum National d'Histoires Naturelles de Paris pour sa colloboration. Enfin, merci aux référés anonymes qui nous auront aidés à améliorer ce manuscrit.

\section{Références citées}

Adjanohoun E. ,1964. Végétation des savanes et des rochers découverts en Côte d'lvoire Centrale. Mém. O.R.S.T.O.M. (7). 209 pp.

Anonyme., 1967. Carte régulière de l'Afrique de l'Ouest à 1/200.000e. République de Côted'Ivoire. I.G.N., Paris.

Anonyme ., 1980. Tableau d'avancement de la cartographie au $1 / 20.000^{\mathrm{e}}$ et au $1 / 50.000 \mathrm{e}$ dela Côte d'Ivoire. Situation au 1er septembre 1978/31 décembre 1979. I.G.N., Paris.

Anonyme., 1983. Ecosystèmes forestiers tropicaux d'Afrique. O.R.S.T.O.M./ U.N.E.S.C.O., Coll. Recherches sur les ressources naturelles, $n^{\circ} 19$, Paris. 473 pp. 
Avenard J. M., 1971. Aspects de la Géomorphologie. In : Avenard J. M., Eldin E., Girard G.,Sircoulon J., Touchebeuf P., Guillaumet J.-L., Adjanohoun E., Perraud A. (eds). Le milieu naturel de la Côte d'lvoire. O.R.S.T.O.M., Paris. p 7-72.

Avenard J. M. \& Tagini B. , 1979. Orohydrographie. A1. Géologie. A2. In : Anonyme (eds). Atlas de Côte d'lvoire. Minist. Plan de Côte d'Ivoire/I.G.T.-Univ.Abidjan/ORSTOM.

Bakayoko A., 1999. Comparaison de la composition floristique et de la structure forestière de parcelles de la Forêt Classée de Bossématié, dans l'Est de la Côte d'Ivoire. D.E.A., U.F.R. Biosciences, Université de Cocody-Abidjan. 72 pp.

Blanc P., 2002. Être plante à l'ombre des forêts tropicales. Nathan/VUEF, Paris, 432 pp.

Chatelain C., Gautier L. \& Spichiger R., 2001. Application du SIG Ivoire à la distribution potentielle des espèces en fonction des facteurs écologiques. Proceedings of the $X V t^{\text {th }}$ AETFAT Congress, Meise (71) : 313-326.

Chevalier A., 1911. Essai d'une carte botanique, forestière et pastorale de l'A.O.F.. C. R. Acad. Sci., 152.

Chevalier A., 1912. Carte botanique, forestière et pastorale de I'A.O.F.. La géographie 26 : 276-277. Ech. 1/3.000.000e, par Erhard Frères, 35 bis rue Denfert Rochereau, Paris.

Chevalier A., 1920. Voyages botaniques. Biogéographie et floristiques générales.

Dabin B., Leneuf N. \& Riou G., 1960. Carte pédologique de la Côte d'Ivoire à 1/2.000.000. Notice explicative.

De Koning J., 1983. La forêt du Banco. I. La forêt. Wageningen Agricultural University, tome I, $156 \mathrm{pp}$.

Eldin M., 1971. Le climat. In : Avenard J. M., Eldin E., Girard G., Sircoulon J., Touchebeuf P., Guillaumet J.-L., Adjanohoun E., Perraud A. (eds). Le milieu naturel de la Côte d'Ivoire. O.R.S.T.O.M., Paris. p 73-108.

Gautier L., Aké Assi L., Chatelain C. \& Spichiger R. 1997. Ivoire : a Geographic Information System for biodiversity management in Ivory Coast. AETFAT, XIVe Congress, Harare, 10 pp.
Guillaumet J.-L., 1967. Recherches sur la végétation et la flore de la région du BasCavally (Côte d'Ivoire). O.R.S.T.O.M., Paris. $247 \mathrm{pp}$.

Guillaumet J.-L., 1979. Végétation. A6a. In : Anonyme (eds). Atlas de Côte d'lvoire. Minist. Plan de Côte d'Ivoire/I.G.T.-Univ. Abidjan/ O.R.S.T.O.M.

Guillaumet J.-L. \& Adjanohoun E., 1968. Carte de la végétation de la Côte d'Ivoire. Ech. 1/ $500.000^{\circ}$, feuille Nord-Ouest, O.R.S.T.O.M., Adiopodoumé, Abidjan.

Guillaumet J.-L. \& Adjanohoun E., 1969. Carte de la végétation de la Côte d'lvoire. Ech. 1/ $500.000^{\mathrm{e}}$, feuilles Sud-Est et Sud-Ouest, O.R.S.T.O.M., Adiopodoumé, Abidjan.

Guillaumet J.-L. \& Adjanohoun E., 1971. La végétation de la Côte d'Ivoire. In : Avenard J. M., Eldin E., Girard G., Sircoulon J., Touchebeuf P., Guillaumet J.-L., Adjanohoun E., Perraud A. (eds). Le milieu naturel de la Côte d'Ivoire. O.R.S.T.O.M., Paris. p 157-266.

Hall J. B. \& Swaine M. D., 1981. Distribution and ecology of vascular plant in a tropical rain forest : forest vegetation in Ghana. Geobotany 1, Dr W. JUNK Publ., The Hague. 383 pp.

Kouamé F. N., 1998. Influence de l'exploitation forestière sur la végétation et la flore de la Forêt Classée du Haut-Sassandra (CentreOuest de la Côte d'lvoire). Thèse Doct. $3^{e}$ Cycle, UFR Biosciences, Université de Cocody-Abidjan. 227 pp.

Kouamé F. N., Kouadio K. E., Kouassi K. \& Poorter L., 2004. Floristic diversity of closed forests in Côte d'Ivoire. In : Poorter L., Bongers F., Kouamé F. N., Hawthorne W. D. (eds). Biodiversity of West African forests. An ecological atlas of woody plant species. CABI Publishing, UK. pp 53-59.

Kouassi K. E., 2000. Contribution à l'étude de la flore de la Forêt Classée de la Haute Dodo, dans le Sud-Ouest de la Côte d'Ivoire, par une approche de relevé de surface. D.E.A., U.F.R. Biosciences, Université de CocodyAbidjan. 102 pp.

Lamotte M. \& Rougerie G., 1952a. Nature et origine du relief de la dorsale guinéenne. $C$. R. Acad. Sci. Paris, t. 235 : 1144-1146. 
Lamotte M. \& Rougerie G., 1952b. Coexistence de trois types de modelés dans les chaînes quartzitiques du Nimba et de Simandou (HauteGuinée française). Ann. Géogr., t. 328, LX1 : 432-442.

Mangenot G., 1955. Etude sur les forêts des plaines et plateaux de la Côte d'Ivoire. Études éburnéennes, Dakar, tome IV : 5-61.

MANGENOT, G., 1956. Les recherches sur la végétation dans les régions tropicales humides de l'Afrique Occidentale. Actes du Colloque de Kandy (Ceylon): l'étude de la végétation tropicale. U.N.E.S.C.O.: 115-126.

Mangenot G., 1971. Une nouvelle carte de la végétation de la Côte d'Ivoire. Mitt. Bot. Staatssamml. München 10 : 116 - 121.

Monnier Y., 1983. Carte de la végétation de la Côte d'Ivoire. In : Vennetier P., Laclavère G. (eds). Atlas de Côte d'lvoire. $2^{\mathrm{e}}$ éd., Jeune Afrique, Paris. $72 \mathrm{pp}$.

Perraud A., 1979. Pédologie. A5a. Aptitudes culturales et forestières des sols. A5b. In : Anonyme (eds). Atlas de Côte d'Ivoire. Minist. Plan de Côte d'Ivoire/I.G.T.-Univ. Abidjan/ O.R.S.T.O.M

Piélou E. C., 1972. Niche width and niche overlap: a method for measuring them. Ecology 53 (4) : 687-692.

Poorter L., Bongers F., Kouamé F. N. \& Hawthorne W. D. 2004. Biodiversity of West African forests. An ecological atlas of woody plant species. CABI publishing, UK. 521 pp.
Richards P. W., 1996. The tropical rain forest. Cambridge University Press, $2^{\mathrm{e}}$ ed. 575 pp.

Riezebos E. P., Vooren A. P., Guillaumet J.-L., Sloot P. H. M. \& Hazeu G. W., 1994. Le Parc National de Taï, Côte d'lvoire. I. Synthèse des connaissances. II. Bibliographie. Tropenbos series 8 , Wageningen. 323 pp. +1 disquette.

Roberty G., 1964. Carte de la végétation de l'Afrique Tropicale Occidentale à l'échelle 1/1.000.000. Introduction et glossaires. O.R.S.T.O.M., Paris.

Rougerie G. \& Lamotte M., 1952. Observations préliminaires sur la chaîne du Nimba. Bull. Ass. Géogr. Fr., $\mathrm{n}^{\circ} 226$ : 113-120.

Sayer J. A., Harcourt C. S. \& Collins N. M., 1992. The conservation Atlas of Tropical forests. Africa. I.U.C.N., Singapore. 288 pp.

Schnell R. (1950). La forêt dense. Introduction à l'étude botanique de la région forestière d'Afrique Occidentale. Lechevalier, Paris. 330 pp.

Ter Braak C. J. F., Smilauer P. (2002). Canoco reference manual and Canodraw for Windows users' guide : software for canonical community ordination (version 4.5). Ithaca, New York. 500 pp.

Trochain J.-L. (1957). Accord interafricain sur la définition des types de végétation de l'Afrique Tropicale. Bulletin de l'Institut des Études Centrafricaines Nouv. Sér. 13/14 : 55-93.

Van Rompaey R. S. A. R. (1993). Forest gradients in West Africa : a spatial gradient analysis. Thèse Doct., Université de Wageningen. $142 \mathrm{pp}$. 
Annexe : Projection des variables sur les axes de l'Analyse Canonique de Correspondance

\begin{tabular}{|c|c|c|c|c|c|}
\hline Sites & Axes & Axe 1 & Axe 2 & Axe 3 & Axe 4 \\
\hline Abngo & & 2,3416 & 0,2056 & 0,2039 & $-0,1335$ \\
\hline Adp1 & & $-0,584$ & $-0,5561$ & 1,6802 & $-1,073$ \\
\hline Adp2 & & $-0,4248$ & $-0,9335$ & $-0,9901$ & $-0,3011$ \\
\hline Adp3 & & $-0,5816$ & 1,888 & $-0,6701$ & $-0,7821$ \\
\hline Adp4 & & $-0,4255$ & 0,4002 & 0,6599 & 1,9411 \\
\hline Agbo & & $-0,0313$ & $-1,054$ & $-1,0734$ & 0,3134 \\
\hline Aym1 & & $-0,2058$ & $-0,1731$ & 0,1555 & $-0,2671$ \\
\hline Aym2 & & $-0,1256$ & $-0,4164$ & 0,2813 & $-0,4684$ \\
\hline Aym3 & & 0,0932 & $-0,2839$ & $-0,1218$ & $-0,2266$ \\
\hline Banco1 & & $-0,2904$ & $-0,325$ & 0,1909 & $-0,5459$ \\
\hline Banco2 & & $-0,2995$ & $-0,2247$ & 0,0269 & $-0,4063$ \\
\hline Banco3 & & $-0,4318$ & $-0,3436$ & 0,6815 & $-0,4348$ \\
\hline Banco4 & & $-0,4767$ & $-0,5469$ & 0,622 & $-0,5172$ \\
\hline Banco5 & & $-0,4076$ & $-0,4855$ & 0,0298 & $-0,5906$ \\
\hline Bsm1 & & $-0,2715$ & 0,2325 & $-0,1287$ & 0,1753 \\
\hline Bsm2 & & $-0,2167$ & 0,2049 & 0,0372 & 0,0878 \\
\hline Cvly & & $-0,0785$ & $-0,2352$ & 0,0761 & $-0,1913$ \\
\hline Dbou & & $-0,1141$ & $-0,0954$ & $-0,2484$ & $-0,0217$ \\
\hline Divo & & 0,7044 & $-0,2678$ & $-0,3756$ & 0,0618 \\
\hline Dnn1 & & 0,3115 & $-0,2418$ & $-0,1627$ & $-0,0661$ \\
\hline Dnn2 & & 0,3973 & $-0,2032$ & $-0,4038$ & $-0,1752$ \\
\hline Frs1 & & $-0,0029$ & $-0,1121$ & $-0,3179$ & $-0,0306$ \\
\hline Frs2 & & 0,0898 & $-0,0878$ & $-0,1814$ & $-0,1716$ \\
\hline Gglo & & 0,2384 & $-0,1167$ & 0,0441 & $-0,2744$ \\
\hline Grb1 & & $-0,2049$ & $-0,3992$ & 0,112 & $-0,1457$ \\
\hline Grb2 & & 0,0522 & $-0,2033$ & $-0,2657$ & $-0,1742$ \\
\hline Hsass1 & & 0,6173 & $-0,2293$ & $-0,3491$ & 0,0058 \\
\hline Hsass2 & & 0,5458 & $-0,1972$ & $-0,2234$ & $-0,1657$ \\
\hline Man1 & & 0,2878 & $-0,2698$ & $-0,6206$ & $-0,4208$ \\
\hline
\end{tabular}


Annexe (Fin) : Projection des variables sur les axes de l'Analyse Canonique de Correspondance

\begin{tabular}{|c|c|c|c|c|}
\hline Axes & Axe 1 & Axe 2 & Axe 3 & Axe 4 \\
\hline Man2 & 0,4315 & 0,0569 & $-0,4203$ & $-0,1226$ \\
\hline Mfre & 0,1198 & $-0,1013$ & $-0,0523$ & $-0,1793$ \\
\hline Monog1 & 0,1745 & $-0,0429$ & $-0,1538$ & $-0,016$ \\
\hline Monog2 & 0,0941 & $-0,2642$ & $-0,3131$ & $-0,1089$ \\
\hline Marah & 0,5121 & 0,0374 & $-0,3193$ & $-0,0884$ \\
\hline Oume & 0,3532 & $-0,2229$ & $-0,3841$ & $-0,2359$ \\
\hline Sbre & 0,2338 & $-0,2175$ & $-0,114$ & $-0,3437$ \\
\hline Tainp1 & $-0,0067$ & 0,0611 & $-0,434$ & $-0,1672$ \\
\hline Tainp2 & 0,1119 & 0,0578 & $-0,4787$ & $-0,1525$ \\
\hline Tainp3 & 0,1691 & $-0,0362$ & $-0,0884$ & $-0,1079$ \\
\hline Tainp4 & 0,2628 & $-0,2085$ & $-0,2674$ & $-0,28$ \\
\hline Tabou & 0,092 & $-0,3702$ & $-0,327$ & $-0,1293$ \\
\hline Yapo1 & 0,0972 & $-0,2442$ & $-0,1363$ & $-0,0292$ \\
\hline Yapo2 & $-0,1548$ & $-0,2233$ & $-0,1935$ & $-0,2654$ \\
\hline Yapo3 & $-0,0322$ & $-0,3462$ & 0,0647 & $-0,2716$ \\
\hline \% de la variabilité & 55,22 & 34,34 & 32,86 & 26,55 \\
\hline
\end{tabular}

